\title{
Low-risk Genes and Multi-organ Cancer Risk in the Polish Population
}

\author{
Tadeusz Dębniak, Cezary Cybulski, Grzegorz Kurzawski, Bohdan Górski, Tomasz Huzarski, Tomasz Byrski, Jacek Gronwald, \\ Janina Suchy, Bartłomiej Masojć, Marek Mierzejewski, Marcin Lener, Urszula Teodorczyk, Krzysztof Mędrek, Elżbieta Złowocka, \\ Ewa Grabowska-Kłujszo, Katarzyna Nej-Wołosiak, Anna Szymańska, Jolanta Szymańska-Pasternak, Joanna Matyjasik, Thierry \\ van de Wetering, Anna Jakubowska, Oleg Oszurek, Aleksandra Tołoczko-Grabarek, Jennifer Castaneda, Rodney Scott, Steven \\ A. Narod, Jan Lubiński
}

International Hereditary Cancer Center, Szczecin, Poland

Key words: CDKN24, CHEK2, NOD2, cancer risk

Corresponding author: Tadeusz Dębniak, International Hereditary Cancer Center, Pomeranian Medical Univeristy, ul. Połabska 4,70-115 Szczecin,Poland; e-mail: debniak@wp.pl

Submitted: 10 December 2005

Accepted: 10 January 2006

There is continuing interest in identifying lowpenetrance genes which are associated with increased susceptibility to common types of cancer. There are several approaches to this problem, including the use of chip-based single nucleotide polymorphism (SNP) arrays to interrogate a large number of genes simultaneously and pre-selecting candidate genes of interest. Candidate genes for cancers of a particular site may be selected because they are known to predispose to malignancies of other organs, or because they are mutated somatically in the cells from the cancer of interest. It is possible that missense variants of genes for which truncating mutations are clearly pathogenic may also be deleterious, but with reduced penetrance. In this situation the association may be overlooked unless large numbers of cancers are studied.

In our centre we performed population-based studies of common variants of three genes: a tumoursuppressor gene CDKN2A (OMIM 600160), NOD2 (OMIM 605956) involved in the chronic inflammation process, and CHEK2 (OMIM 604373) participating in the DNA damage response.

To determine whether CDKN2A common variant A $148 \mathrm{~T}$ may be associated with an increased risk of malignancies at different sites of origin we genotyped a series of 8,263 unselected cancer cases and compared the frequency of the change observed in this population to 3,000 controls in Poland. To establish the range of cancer types associated with three CHEK2 mutations (VS2 + 1G $\rightarrow$ A, 1100 delC, and I157T) we genotyped 4,008 unselected cases of cancer and 4,000 controls in Poland. In order to define the range of cancer phenotypes associated with the NOD2 3020ins C mutation we examined 2,604 unselected invasive cancers of 12 different types and 1,910 controls from Poland.

\section{Results}

We showed an association between CDKN2A common variant and increased risk of malignant melanoma $(O R=2.1)$, cancers of breast (under 50y, $\mathrm{OR}=1.5$ ), lung $(\mathrm{OR}=2.0$ ) and colon $(\mathrm{OR}=1.5)$ (table 1) [1-3].

We also found a positive association between common NOD2 variant and cancers of the colon (lateonset, $O R=2.2$ ), breast (early-onset breast cancer $\mathrm{OR}=1.9$ and ductal breast cancer with an in situ component $O R=2.1$ ) and ovary (table 2) [4-7].

Positive associations with CHEK2 protein-truncating alleles were seen for cancers of the thyroid $(O R=4.9)$, breast $(O R=2.2)$ and prostate $(O R=2.2)$. The missense variant 1157T was associated with an increased risk of breast cancer $(O R=1.4)$, colon cancer $(O R=2.0)$, kidney cancer $(O R=2.1)$, prostate cancer $(O R=1.7)$ and thyroid cancer (OR=1.9) (table 3) [8]. 
Table 1. Association between A148T variants and selected types of cancer

\begin{tabular}{|c|c|c|c|c|c|}
\hline \multicolumn{3}{|c|}{$\mathrm{A} 148 \mathrm{~T}$} & \multirow[t]{2}{*}{ OR } & \multirow[t]{2}{*}{$95 \%$ Confidence Interval } & \multirow[t]{2}{*}{$\mathrm{p}$ (adjusted $\mathrm{p}$ ) } \\
\hline total controls $(n=3000)$ & $105(3.5 \%)$ & & & & \\
\hline bladder ( $n=223$ ) & $0(0 \%) \mathrm{A} / \mathrm{A}$ & 7 (3.1\%) G/A & 0.9 & $0.4105-1.945$ & 0.7764 (n.s) \\
\hline colon $(n=724)$ & $0(0 \%) \mathrm{A} / \mathrm{A}$ & $37(5.1 \%) \mathrm{G} / \mathrm{A}$ & 1.5 & $1.012-2.180$ & $0.0423(0.5499)$ \\
\hline stomach $(n=246)$ & $0(0 \%) \mathrm{A} / \mathrm{A}$ & 8 (3.3\%) G/A & 0.9 & $0.4461-1.925$ & 0.8384 (n.s) \\
\hline larynx $(n=396)$ & $0(0 \%) \mathrm{A} / \mathrm{A}$ & 17 (4.3\%) G/A & 1.2 & $0.7326-2.088$ & 0.4255 (n.s) \\
\hline ovary $(n=340)$ & $0(0 \%) \mathrm{A} / \mathrm{A}$ & $12(3.5 \%) \mathrm{G} / \mathrm{A}$ & 1.0 & $0.5491-1.853$ & 0.9777 (n.s) \\
\hline lung $(n=497)$ & $0(0 \%) \mathrm{A} / \mathrm{A}$ & $34(6.8 \%) \mathrm{G} / \mathrm{A}$ & 2.0 & $1.358-3.018$ & $0.0004(0.0052)$ \\
\hline prostate $(n=348)$ & $0(0 \%) \mathrm{A} / \mathrm{A}$ & 13 (3.7\%) G/A & 1.1 & $0.5946-1.925$ & 0.8215 (n.s) \\
\hline kidney $(n=264)$ & $0(0 \%) \mathrm{A} / \mathrm{A}$ & $6(2.3 \%) \mathrm{G} / \mathrm{A}$ & 0.6 & $0.2788-1.474$ & 0.2915 (n.s) \\
\hline thyroid $(n=173)$ & $0(0 \%) \mathrm{A} / \mathrm{A}$ & $3(1.7 \%) \mathrm{G} / \mathrm{A}$ & 0.5 & $0.1528-1.549$ & 0.2129 (n.s) \\
\hline $\begin{array}{l}\text { non-Hodgkin } \\
\text { Lymphoma }(n=162)\end{array}$ & $0(0 \%) \mathrm{A} / \mathrm{A}$ & $6(3.7 \%) \mathrm{G} / \mathrm{A}$ & 1.1 & $0.4585-2.453$ & 0.8909 (n.s) \\
\hline $\begin{array}{l}\text { breast (under 50y) } \\
(n=3318)\end{array}$ & $0(0 \%) \mathrm{A} / \mathrm{A}$ & 168 (5.1\%) G/A & 1.5 & $1.2764-1.832$ & 0.002 \\
\hline melanoma $(n=471)$ & $0(0 \%) \mathrm{A} / \mathrm{A}$ & $33(7 \%) \mathrm{G} / \mathrm{A}$ & 2.1 & $1.387-3.111$ & 0.0003 \\
\hline pancreas $(n=210)$ & $0(0 \%) \mathrm{A} / \mathrm{A}$ & $8(3.8 \%) \mathrm{G} / \mathrm{A}$ & 1.1 & $0.5246-2.273$ & 0.8140 (n.s) \\
\hline
\end{tabular}

Table 2. Association of the NOD2 3020insC mutation and selected types of cancer

\begin{tabular}{|c|c|c|c|c|c|}
\hline Site & Number tested & Number positive & Prevalence of 3020 ins C (\%) & Odds ratio & $\mathrm{p}$-value \\
\hline bladder & 172 & 18 & 10.5 & 1.5 & 0.13 \\
\hline breast & 462 & 37 & 8.0 & 1.1 & 0.62 \\
\hline with DCIS & 126 & 18 & 14.3 & 2.1 & 0.009 \\
\hline without DCIS & 336 & 19 & 5.7 & 0.76 & 0.30 \\
\hline colon & 255 & 31 & 12.2 & 1.8 & 0.01 \\
\hline kidney & 245 & 8 & 3.2 & 0.4 & 0.02 \\
\hline larynx & 223 & 23 & 10.3 & 1.5 & 0.11 \\
\hline lung & 258 & 30 & 11.6 & 1.7 & 0.03 \\
\hline melanoma & 198 & 10 & 5.1 & 0.7 & 0.31 \\
\hline ovary & 317 & 35 & 11.0 & 1.6 & 0.03 \\
\hline pancreas & 127 & 6 & 4.7 & 0.6 & 0.37 \\
\hline prostate & 298 & 17 & 5.7 & 0.76 & 0.40 \\
\hline stomach & 213 & 20 & 9.4 & 1.3 & 0.27 \\
\hline thyroid & 82 & 8 & 9.8 & 1.4 & 0.39 \\
\hline controls & 1910 & 140 & 7.3 & & \\
\hline
\end{tabular}


Table 3. Association between CHEK2 variants and selected types of cancer

\begin{tabular}{|c|c|c|c|c|c|}
\hline \multirow[t]{2}{*}{ Site } & \multirow[t]{2}{*}{ No. tested } & \multicolumn{4}{|c|}{ Number positive (prevalence), odds ratio, p-value } \\
\hline & & $\mathrm{IVS} 2+1 \mathrm{G}>\mathrm{A}$ & 1100delC & Any truncating mutation & $1157 \mathrm{~T}$ \\
\hline \multirow[t]{2}{*}{ bladder } & 172 & $1(0.6 \%)$ & & $1(0.6 \%)$ & $12(7.0 \%)$ \\
\hline & & OR $1.2 p=0.7$ & 0 & OR $0.8 p=0.8$ & OR $1.5 p=0.3$ \\
\hline \multirow[t]{2}{*}{ breast } & 1017 & $11(1.1 \%)$ & $5(0.5 \%)$ & $16(1.6 \%)$ & $68(6.7 \%)$ \\
\hline & & OR $2.3 p=0.04$ & OR $2.0 p=0.3$ & OR $2.2 p=0.02$ & OR $1.4 p=0.02$ \\
\hline \multirow[t]{2}{*}{ colon } & 300 & $1(0.3 \%)$ & $2(0.7 \%)$ & $3(1 \%)$ & $28(9.3 \%)$ \\
\hline & & OR $0.7 p=0.9$ & OR $2.7 p=0.4$ & OR $1.4 \quad p=0.8$ & OR $2.0 p=0.001$ \\
\hline \multirow[t]{2}{*}{ kidney } & 264 & 0 & $2(0.8 \%)$ & $2(0.8 \%)$ & $26(9.8 \%)$ \\
\hline & & & OR $2.7 p=0.5$ & OR $1.0 \quad p=0.8$ & OR $2.1 p=0.0006$ \\
\hline \multirow[t]{2}{*}{ larynx } & 245 & 0 & 0 & 0 & $10(4.1 \%)$ \\
\hline & & & & & OR $0.8 p=0.7$ \\
\hline \multirow[t]{2}{*}{ lung } & 272 & 0 & 0 & 0 & $7(2.6 \%)$ \\
\hline & & & & & OR $0.5 \quad p=0.1$ \\
\hline \multirow[t]{2}{*}{ melanoma } & a 129 & $2(1.5 \%)$ & $1(0.8 \%)$ & $3(2.3 \%)$ & $6(4.6 \%)$ \\
\hline & & OR $3.3 p=0.3$ & OR $3.1 p=0.8$ & OR $3.2 p=0.1$ & OR $1.0 p=0.9$ \\
\hline \multirow[t]{2}{*}{ ovary } & 292 & 0 & 0 & 0 & $14(4.8 \%)$ \\
\hline & & & & & OR $1.0 p=0.9$ \\
\hline \multirow[t]{2}{*}{ prostate } & 690 & $8(1.2 \%)$ & $3(0.4 \%)$ & 11 (1.6\%) & $54(7.8 \%)$ \\
\hline & & OR $2.5 \quad p=0.05$ & OR $1.7 p=0.2$ & OR $2.2 p=0.04$ & OR $1.7 p=0.002$ \\
\hline \multirow[t]{2}{*}{ stomach } & 241 & $4(1.7 \%)$ & 0 & $4(2.1 \%)$ & $13(5.4 \%)$ \\
\hline & & OR $3.5 \quad p=0.05$ & & OR $2.3 p=0.2$ & OR $1.1 p=0.8$ \\
\hline \multirow[t]{2}{*}{$\mathrm{NHL}$} & 120 & $1(0.8 \%)$ & 0 & $1(0.8 \%)$ & 11 (9.2\%) \\
\hline & & OR $1.8 p=0.9$ & & OR $1.1 \quad p=0.7$ & OR $2.0 \quad p=0.05$ \\
\hline \multirow[t]{2}{*}{ pancreas } & 93 & 0 & 0 & 0 & $6(6.4 \%)$ \\
\hline & & & & & OR $1.4 p=0.6$ \\
\hline \multirow[t]{2}{*}{ thyroid } & 173 & $5(2.9 \%)$ & $1(0.6 \%)$ & $6(3.5 \%)$ & $15(8.7 \%)$ \\
\hline & & OR $6.2 p=0.0003$ & OR $2.3 p=0.9$ & OR $4.9 p=0.0006$ & OR $1.9 p=0.04$ \\
\hline controls & 4000 & $19(0.475 \%)$ & $10(0.25 \%)$ & $29(0.725 \%)$ & $193(4.825 \%)$ \\
\hline
\end{tabular}

\section{Conclusions}

It seems that CDKN2A, NOD2 and CHEK2 are responsible for a wide range of cancer types.

We estimate that the mutations mentioned above are responsible for around $20 \%$ of malignancies occurring in the Polish population. According to our studies over 4 million people in Poland carry one of the mutations described above. We elaborated genetic tests for CDKN2A, NOD2 and CHEK2 aimed at reliable identification of persons with increased risk of developing cancers of the breast, ovary, lung, prostate, thyroid, colon, kidney and malignant melanoma.

\section{References}

1. Debniak T, Scott RJ, Huzarski T, Byrski T, Rozmiarek A, Debniak B, Gorski B,Cybulski C, Medrek K, Mierzejewski M, Masojc B, Matyjasik J, Zlowocka E,Teodorczyk U, Lener M, KlujszoGrabowska E, Nej-Wolosiak K, Jaworowska E, Oszutowska D, Szymanska A, Szymanska J, Castaneda J, van de Wetering T, Suchy J, Kurzawski G, Oszurek $O$, Narod S and Lubinski J. CDKN2A common variant and multi-organ cancer risk-a population-based study. Int J Cancer 2006 (in press).

2. Debniak T, Gorski B, Huzarski T, Byrski T, Cybulski C, Mackiewicz A, Gozdecka-Grodecka S, Gronwald J, Kowalska E, Haus O, Grzybowska E, Stawicka M, Swiec M, Urbanski K, Niepsuj S, Wasko B, Gozdz S, Wandzel P, Szczylik C, Surdyka D, Rozmiarek A, Zambrano O, Posmyk M, Narod SA and Lubinski J. A 
common variant of CDKN2A (p16) predisposes to breast cancer. J Med Genet 2005; 42: 763-765.

3. Debniak T, Scott RJ, Huzarski T, Byrski T, Rozmiarek A, Debniak B, Zaluga E, Maleszka R, Kladny J, Gorski B, Cybulski C, Gronwald J, Kurzawski $G$ and Lubinski J. CDKN2A common variants and their association with melanoma risk: a populationbased study. Cancer Res 2005; 65: 835-839.

4. Lener MR, Oszutowska D, Castaneda J, Kurzawski G, Suchy J, Nej-Wolosiak K, Byrski T, Huzarski T, Gronwald J, Szymanska A, Szymanska-Pasternak J, Grodzki T, Serwatowski P, Bre Borowicz G, Scott RJ and Lubinski J. Prevalence of the NOD2 3020ins $C$ mutation in aggregations of breast and lung cancer. Breast Cancer Res Treat 2005 Nov 3; 1-5.

5. Huzarski T, Lener M, Domagala W, Gronwald J, Byrski $T$, Kurzawski G, Suchy J, Chosia M, Woyton J, Ucinski M, Narod SA and Lubinski J. The 3020insC allele of NOD2 predisposes to early-onset breast cancer. Breast Cancer Res Treat 2005; 89: 91-93.

6. Kurzawski G, Suchy J, Kladny J, Grabowska E, Mierzejewski M, Jakubowska A, Debniak T, Cybulski C, Kowalska E, Szych Z, Domagala W, Scott RJ and Lubinski J. The NOD2 3020insC mutation and the risk of colorectal cancer. Cancer Res 2004; 64: 1604-1606

7. Lubinski J, Huzarski T, Kurzawski G, Suchy J, Masoic B, Mierzejewski M, Lener M, Domagala W, Chosia M, Teodorczyk U, Medrek K, Debniak T, Złowocka E, Gronwald J, Byrski T, Grabowska E, Nej K, Szymanska A, Szymanska J, Matyjasik J, Cybulski C, Jakubowska A, Gorski B and Narod SA. The 3020insC Allele of NOD2 Predisposes to Cancers of Multiple Organs. Her Can in Clin Pract 2005; 3: 59-63.

8 Cybulski C, Gorski B, Huzarski T, Masojc B, Mierzejewski M, Debniak T, Teodorczyk U, Byrski T, Gronwald J, Matyjasik J, Zlowocka E, Lenner M, Grabowska E, Nej K, Castaneda J, Medrek K, Szymanska A, Szymanska J, Kurzawski G, Suchy J, Oszurek O, Witek A, Narod SA and Lubinski J. CHEK2 is a multiorgan cancer susceptibility gene. Am J Hum Genet 2004; 75: 1131-1135. 